\title{
Modelling analysis of surface stress on a rectangular cantilever beam
}

\author{
Yin Zhang, Quan Ren and Ya-pu Zhao \\ State Key Laboratory of Nonlinear Mechanics (LNM), Institute of Mechanics, \\ Chinese Academy of Sciences, Beijing 100080, People's Republic of China \\ E-mail: yzhao@lnm.imech.ac.cn
}

Received 14 April 2004

Published 14 July 2004

Online at stacks.iop.org/JPhysD/37/2140

doi:10.1088/0022-3727/37/15/014

\begin{abstract}
Three models of surface stress on a rectangular cantilever beam are presented. The surface stress is modelled as a corresponding concentrated moment at the beam free end, a corresponding concentrated moment plus a corresponding concentrated axial load at the beam free end, and a corresponding uniformly distributed axial stress plus bending moment per unit length along the beam span, respectively. The results of the three models are compared under three different loading scenarios. We also present an analysis of the error source, when using Stoney's formula to predict the surface stress, by comparing the kinematic and loading assumptions of the three models. The surface stress effects on structure deflection are usually modelled as bending moments applied at structure free edge(s)/end(s). Modelling the surface stress effect along the beam neutral axis is presented and compared with modelling its effect at free edge(s)/end(s). The stiffening effect of tensile surface stress is also studied.
\end{abstract}

\section{Introduction}

The sources of surface stress can be the surface reconstruction [1], or the interactions between a surface and adsorbates [2-6], or the interactions between a surface and its ambient environment [7,8] etc. In the size domain of microelectromechanical systems (MEMS), surface stresses scale linearly with dimension $[9,10]$ and surface to volume ratio increases as microstructure scale decreases [11]. For these reasons, surface stress can be overwhelming and very important in microstructures. Surface stress can induce selfassembly [9] or be induced by self-assembly [5]. Surface stress changes of DNA hybridization and receptor-ligand binding induce the differential deflection of the cantilever sensor, which can be used for a molecular recognition mechanism [6]. Surface stress has been studied extensively and Stoney's formula [12] serves as a cornerstone for curvature based analysis and a technique for the measurement of surface stress [13-15]. The very essence of Stoney's formula is to assume a uniform curvature for the whole deflected structure and make a linear analysis of small deflection. For structures under larger deflection or 'thick' film/coating on the substrate, Stoney's formula does not agree well with the experimental data [16,17].
Klein [16] and Freund et al [17] provided revised forms of Stoney's formula by considering the effects of the ratio of the Young's modulus/thickness of the film/coating to the substrate's Young's modulus/thickness. Using linear analysis, both Klein and Freund et al conclude that Stoney's formula holds for the structure with a large ratio of substrate thickness to film/coating thickness, i.e. 'thin' film case. Although they point out that the kinematic assumption of uniform curvature for the nonlinear analysis of film large deflection is quite extreme and they also illustrate its limitation, Freund et al [17] still use uniform curvature as the kinematic assumption. The problem of uniform curvature is solved by enforcing the system's potential energy to be stationary for equilibrium. In this paper, there is no such kinematic assumption of uniform curvature. We also demonstrate under what modelling conditions, the uniform curvature kinematic assumption is valid for the cantilever beam structure. Another characteristic of Stoney's formula is to model the surface stress as a moment applied at the structure's free end. Sader [18] concludes that the application of a differential surface stress on the plate is equivalent to the loading of the free edges by the corresponding moment per unit length. Godin et al [19] and Miyatani and Fujihira [20] measure surface stress by comparing the deflections of the 
cantilever beam due to the concentrated transverse load and the moment at the cantilever free end, respectively. All these analyses that model the surface stress as a moment applied at the structure's free edge(s)/end(s) do not take into account the influence of the surface stress on structure stiffness. It is also impossible to use these models to explain the local bending mode due to surface stress [21].

This paper aims to offer a modelling analysis that explains the disagreement between the experimental data and theoretical prediction of Stoney's formula reported by Klein and Freund et al $[16,17]$. Also, model \#3 in this paper can explain the local bending phenomenon reported by Liu et al [21] if we model the surface stress distribution as a local stressor [21]. In MEMS structures, the surface stress or stress gradient is computed by measuring the curvature or displacement of the structure rather than measuring it directly $[19,20,22]$. In this paper, it is quite clear that we show that under different modelling of surface stress influence on the structure, the structure generates different restoring forces by deflecting/deforming differently to counteract the surface stress to achieve the equilibrium. Three models on the surface stress effects are examined and compared in this paper. We demonstrate that the stiffening effect of tensile surface stress becomes very important when the magnitude of the surface stress is relatively large. Therefore, the modelling analysis will be very important for evaluating surface stresses/stress gradients in the (MEMS) structure.

\section{Model development}

In figure $1(a)$, the beam tip is under a uniformly distributed loading, $\sigma$. Its unit is Newton/metre, not pascal and $\sigma>0$ is tensile. For historical reasons, we call $\sigma$ the surface stress. This surface stress effect is modelled as a concentrated moment $M$ applied at the cantilever beam's free end. Thus, the governing equation of the beam deflection for this model (model \#1): is as follows:

$$
E^{*} I \frac{\mathrm{d}^{2} y}{\mathrm{~d} x^{2}}=M
$$

and the boundary conditions are given as

$$
y(0)=0, \quad \frac{\mathrm{d} y}{\mathrm{~d} x}(0)=0 .
$$

Here $E^{*}$ is the biaxial modulus $[16,17,23] . E^{*}$ is defined as $E^{*}=E /(1-\mu) . \quad E$ is the beam Young's modulus, $\mu$ the
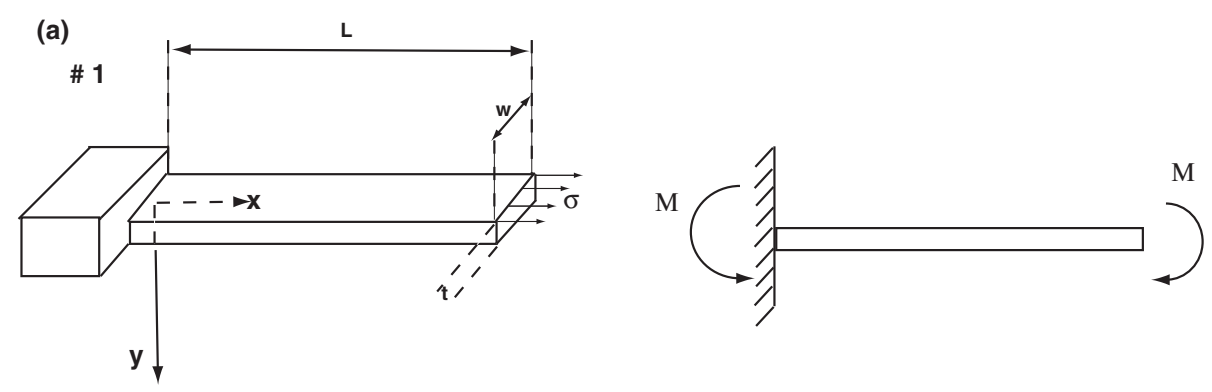

(b)

\# 2
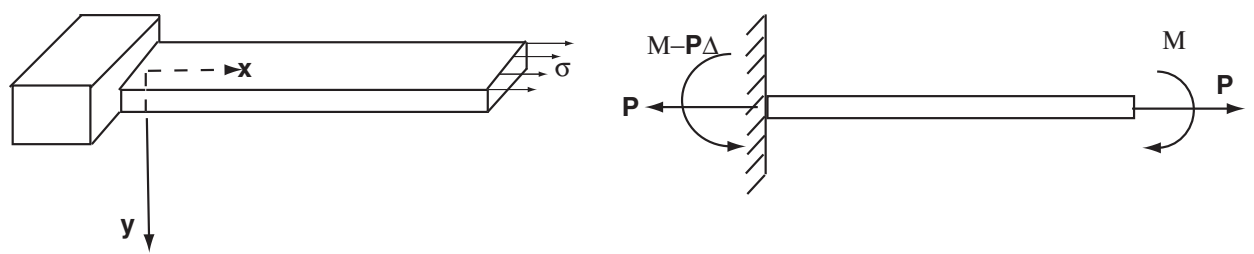

(c)

\# 3
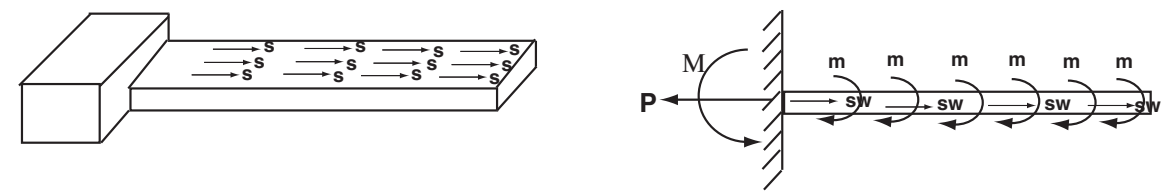

Figure 1. Schematic diagram of the cantilever beam dimensions, coordinates and the loading scenarios of the three models. 
Poisson's ratio, $y$ the beam deflection and $I$ the area moment of inertia. For a rectangular beam, $I=w t^{3} / 12, w$ and $t$ are the beam width and thickness, respectively, $M$ is the concentrated moment defined as $M=\sigma w t / 2$. Equation (1) is solved by using integration and boundary conditions as follows:

$$
y=\frac{M x^{2}}{2 E^{*} I} .
$$

For linear analysis of small deflection, the curvature $\kappa$ of the deflected beam is approximated as $\mathrm{d}^{2} y / \mathrm{d} x^{2}$. So for model \#1, the curvature $\kappa$ and the radius of curvature $R$ are given as

$$
\kappa=\frac{1}{R}=\frac{M}{E^{*} I}=\frac{6 \sigma}{E^{*} t^{2}} .
$$

The equation above is the modified Stoney's formula (in the original Stoney's formula, $E^{*}$ is substituted by $E$ ) [20]. In this case, we assume surface stress exists only on the beam's upper surface, for the sake of clarity and simplicity. For more general cases, $\sigma$ is substituted by differential surface stress $\Delta \sigma=\sigma^{+}-\sigma^{-} . \sigma^{+}$and $\sigma^{-}$are surface stresses on the upper and lower surfaces, respectively. Also, this substitution does not affect any derivation for linear analysis. Clearly, the modelling of the surface stress effect as a concentrated moment at the cantilever beam's free end guarantees that the beam curvature is uniform (constant).

Model \#2 is shown in figure $1(b)$. The surface stress effect is modelled as a corresponding concentrated bending moment $M$ plus a corresponding concentrated axial load $P$ at the beam's free end. $M$ is also defined as $M=\sigma w t / 2$ and $P=\sigma w . P>0$ is tensile. For reasons of simplicity and brevity, we omit the derivation of the governing equation and its boundary conditions for this model, and directly give the governing equation as

$$
E^{*} I \frac{\mathrm{d}^{4} y}{\mathrm{~d} x^{4}}-P \frac{\mathrm{d}^{2} y}{\mathrm{~d} x^{2}}=0
$$

and boundary conditions as

$$
\begin{gathered}
y(0)=0, \quad \frac{\mathrm{d} y}{\mathrm{~d} x}(0)=0, \quad E^{*} I \frac{\mathrm{d}^{2} y}{\mathrm{~d} x^{2}}(L)=M \\
E^{*} I \frac{\mathrm{d}^{3} y}{\mathrm{~d} x^{3}}(L)=P \frac{\mathrm{d} y}{\mathrm{~d} x}(L) .
\end{gathered}
$$

Here, $L$ is the beam length. To non-dimensionalize equations (5) and (6), the following dimensionless variables are introduced:

$$
\xi=\frac{x}{L}, \quad Y=\frac{y}{L} .
$$

Equations (5) and (6) are now non-dimensionalized as

$$
Y^{\prime \prime \prime \prime}-(\beta L)^{2} Y^{\prime \prime}=0
$$

and the boundary conditions are

$$
\begin{aligned}
Y(0)=0, \quad Y^{\prime}(0) & =0, \quad Y^{\prime \prime}(1)=\frac{\alpha(\beta L)^{2}}{2}, \\
Y^{\prime \prime \prime}(1) & =(\beta L)^{2} Y^{\prime}(1) .
\end{aligned}
$$

Here ()$^{\prime}=\mathrm{d} / \mathrm{d} \xi, \beta=P / E^{*} I$ and $\alpha=t / L$. Equation (5) is the Euler-Bernoulli beam model, which ignores the shear force effect on the beam deflection. $\alpha$ is thus prescribed as a small number, which physically means that the model is only valid for thin beams.

Equation (7) can also be solved by integration and using boundary conditions. The solution is given as

$$
Y=\frac{\alpha}{2 \cosh (\beta L)}[\cosh (\beta L \xi)-1] .
$$

The curvature $\Pi$ of $Y$ is given as

$$
\Pi=Y^{\prime \prime}=\frac{\alpha(\beta L)^{2}}{2 \cosh (\beta L)} \cosh (\beta L \xi) .
$$

Clearly, from this equation, the curvature $\Pi$ is not a constant along the beam span in model \#2.

Equations (3) and (4) of model \#1 are also nondimensionalized as

$$
Y=\frac{\alpha(\beta L)^{2} \xi^{2}}{4}
$$

and

$$
\Pi=Y^{\prime \prime}=\frac{\alpha(\beta L)^{2}}{2} .
$$

For model \#3, there is an area stress $s$ uniformly distributed on the upper surface of the beam. The unit of $s$ is pascal. We model this as a uniformly distributed axial stress $s w$ along the beam's neutral axis and a uniformly distributed bending moment $m$ along the beam. $m$ is defined as $m=s w t / 2$. For this model, it is not that easy to obtain the governing equation as for the previous two. The principle of virtual work (PVW) is used here to derive the governing equation and its boundary conditions. The elastic bending energy of the beam is given as

$$
V=\int_{0}^{L} \frac{E^{*} I}{2}\left(\frac{\mathrm{d}^{2} y}{\mathrm{~d} x^{2}}\right)^{2} \mathrm{~d} x .
$$

The external work done by the uniformly distributed moment $m$ and $s w$ is

$$
W=\int_{0}^{L} m(L-x) \frac{\mathrm{d}^{2} y}{\mathrm{~d} x^{2}} \mathrm{~d} x-\int_{0}^{L} \frac{1}{2} s w(L-x)\left(\frac{\mathrm{d} y}{\mathrm{~d} x}\right)^{2} \mathrm{~d} x .
$$

By applying PVW, $\delta(V-W)=0$, the governing equation and boundary conditions are derived as

$$
E^{*} I \frac{\mathrm{d}^{4} y}{\mathrm{~d} x^{4}}-s w(L-x) \frac{\mathrm{d}^{2} y}{\mathrm{~d} x^{2}}+s w \frac{\mathrm{d} y}{\mathrm{~d} x}=0
$$

and the boundary conditions are

$$
\begin{gathered}
y(0)=0, \quad \frac{\mathrm{d} y}{\mathrm{~d} x}(0)=0, \quad \frac{\mathrm{d}^{2} y}{\mathrm{~d} x^{2}}(L)=0, \\
E^{*} I \frac{\mathrm{d}^{3} y}{\mathrm{~d} x^{3}}(L)+m=0 .
\end{gathered}
$$

It can be seen that these boundary conditions are quite different from the boundary conditions of model \#2 in equation (6). Freund et al [17] incorporate the mismatch strain due to the surface stress into the film's elastic potential energy, which is physically equivalent to adding a uniformly distributed load $s w$ and bending moment $m$ into the elastic system. In reality, 
model \#3 models the real surface stress effect better. Here, $s w$ and $m$ are uniformly distributed along the whole beam. By adjusting the distribution of $s w$ and $m$, model \#3 is able to provide an explanation for the local bending mode reported by Liu et al [21].

The two equations above are also non-dimensionalized as

$$
Y^{\prime \prime \prime \prime}-(\beta L)^{2}(1-\xi) Y^{\prime \prime}+(\beta L)^{2} Y^{\prime}=0
$$

and

$$
\begin{gathered}
Y(0)=0, \quad Y^{\prime}(0)=0, \quad Y^{\prime \prime}(1)=0, \\
Y^{\prime \prime \prime}(1)+\frac{\alpha(\beta L)^{2}}{2}=0 .
\end{gathered}
$$

Because the variable $\xi$ appears in equation (17), its analytical solution will be very difficult to obtain, if not impossible. The numerical method, the shooting method for two point boundary value problems [24] is applied here to solve equation (17) with its boundary conditions.

In order to compare these three models, we define that $s$, $\sigma, P, m$ and $M$ have the following relations

$$
\begin{gathered}
\sigma=s L, \quad P=\sigma w=s L w, \quad m=\frac{s w t}{2}, \\
M=\frac{\sigma w t}{2}=\frac{s L w t}{2}=m L .
\end{gathered}
$$

\section{Results and discussions}

For the Euler-Bernoulli beam model, in all the three cases shown here, $\alpha$ is set to be a small number, 0.05. Figure 2 shows the deflections computed by these three models at a small load of $\beta L=0.1$. There is almost no difference between models \#1 and \#2. The stiffening effect due to the tensile load $P$ in model \#2 is not obvious for this small loading scenario. The reason for the smaller deflection computed by model \#3 is that for models \#1 and \#2, the surface stress is modelled as a concentrated moment at the beam's free end,

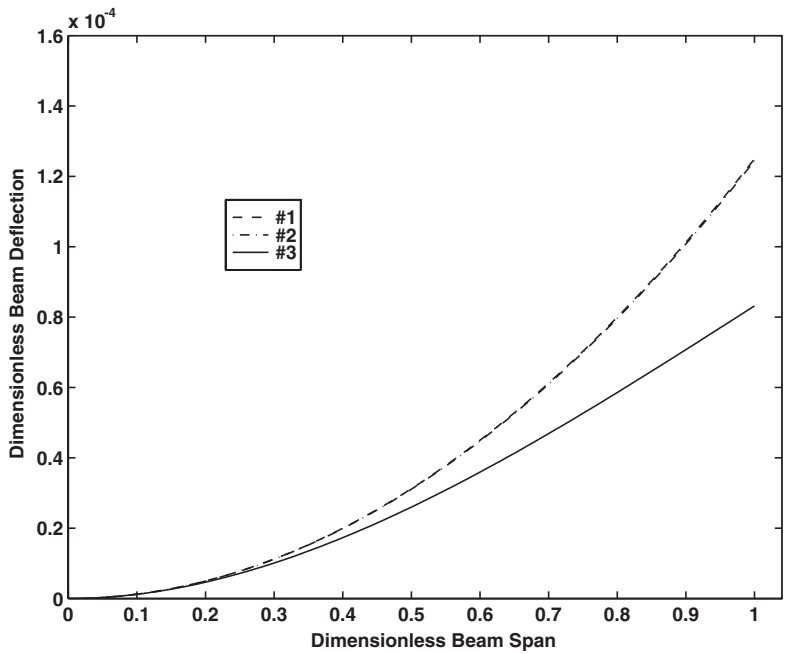

Figure 2. A comparison of the deflections computed from the three different models under small loading scenario at $t / L=0.05$ and $\beta L=0.1$. which maximizes the surface stress bending effect, while for model \#3, the bending moment is uniformly distributed along the beam span. Figure 3 shows the curvatures $\left(Y^{\prime \prime}\right)$ computed by these three models at $\beta L=0.1$. It also shows that there is almost no difference between models \#1 and \#2, whereas for model \#3, the curvature of model \#3 starts at the same point as that of model \#1, and then decreases linearly to zero. In linear analysis, the curvature is directly related to the bending moment. For model \#3, the bending contribution of axial stress $(s w)$ to the cantilever's fixed end, which is $\int_{0}^{L}(s w y / 2) \mathrm{d} x$, is ignored. This is reflected in the external work expression in equation (14). To keep this axial stress bending moment contribution, $\int_{0}^{L}(s w y / 2) \mathrm{d} x$, equation (15) will become an integral equation. For small deflection $y$, this contribution is very small compared with $m L$. Therefore, the bending moment at the beam's fixed end of model \# 3 is the same as that of model \#1. This is the reason why the curvature of model \#3 has the same starting point as that of model \#1 at the beam's fixed end. The third boundary condition in equation (16) requires the curvature of model \#3 to be zero at the beam's tip.

Figure 4 shows the comparison of the deflections computed by the three models for the $\beta L=1$ case. Now the deflection of model \#2 is obviously smaller than that of model \#1. This is due to the stiffening effect of the tensile force $P$ at the beam's free end. This tensile force's stiffening effect is also reflected in the frequency domain [25]. Figure 5 shows the curvature comparison of the three models at $\beta L=1$. Clearly, the curvature of model \#2 starts below that of model \#1 at the beam's fixed end and ends at the same value at the beam tip. For model \#1, the bending moment at the beam's fixed end is $M$, while for model \#2, the bending moment is $M-P \Delta . \Delta=y(L)$ is the beam tip's deflection. That is why the curvature of model \#2 starts at a lower value, and the third boundary condition in equation (6) enforces the curvature of model \#2 to end with the same value as that of model \#1.

Figure 6 shows the comparison of the deflections computed by the three models for the case $\beta L=4$. Clearly, the deflection of model \#3 is larger than that of model \#2. As mentioned above, model \#2 has the advantage

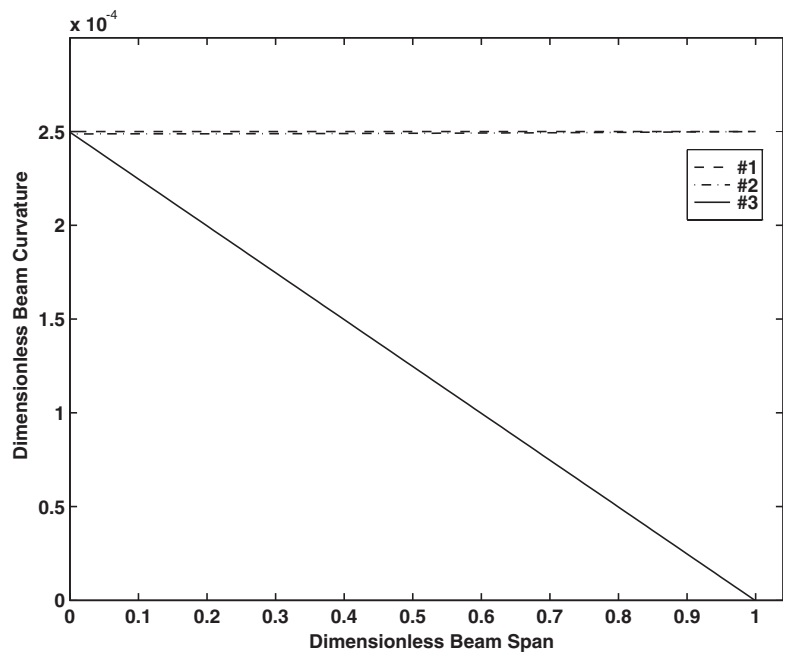

Figure 3. A comparison of the curvatures computed from the three different models under small loading scenario at $t / L=0.05$ and $\beta L=0.1$. 


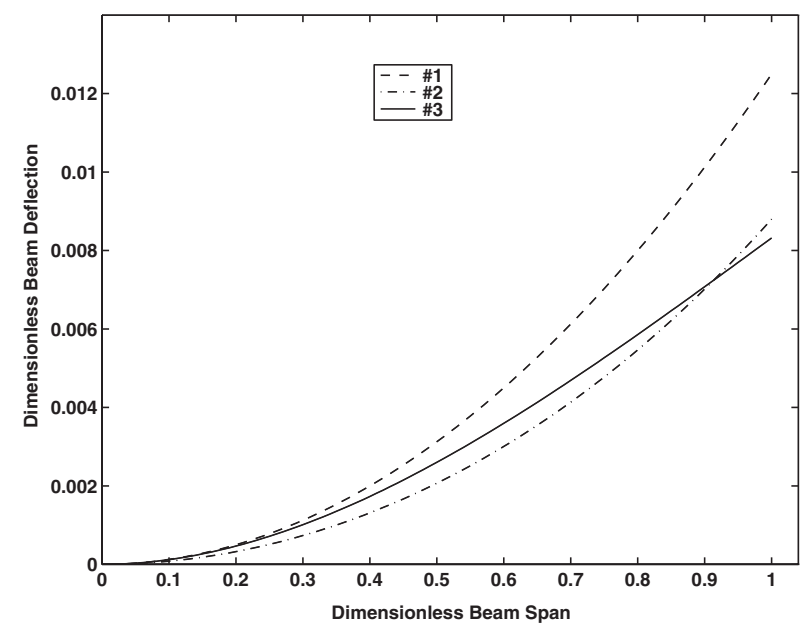

Figure 4. A comparison of the deflections computed from the three different models under medium loading scenario at $t / L=0.05$ and $\beta L=1$.

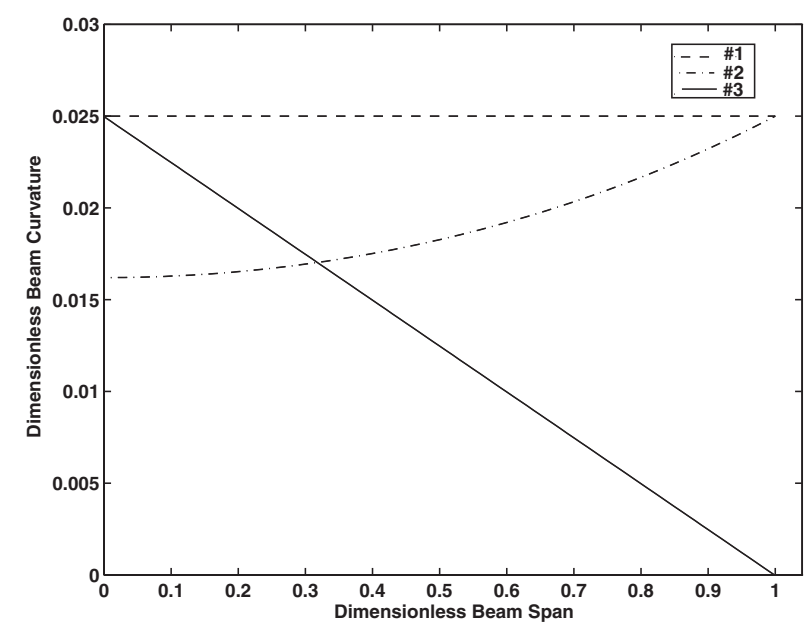

Figure 5. A comparison of the curvatures computed from the three different models under medium loading scenario at $t / L=0.05$ and $\beta L=1$.

of maximizing the bending effect over model \#1 by modelling a concentrated moment at the beam's free end. The reason why model \#3 has a larger deflection than that of model \#2 lies in the fact that uniformly distributed tensile axial loads $(s w)$ and tensile concentrated axial loads $(P)$ have quite different stiffening effects on the structure. For a cantilever beam structure, as Timoshenko [26] demonstrates (approximately), the effect on the frequency of uniformly distributed tensile forces is the same as if $\frac{7}{20}$ of the sum of these forces are applied at the end of the beam. A tensile concentrated axial load has a much larger stiffening effect than a uniformly distributed tensile axial load. Figure 7 shows the curvature comparison of the three models at $\beta L=4$. Clearly the curvature difference at the beam's fixed end between model \#2 and models \#1 and \#3 enlarges because the beam tip deflection increases under this larger loading scenario.

\section{Conclusion}

The Euler-Bernoulli beam model is used to analyse the small deflection due to the surface stresses. Three different

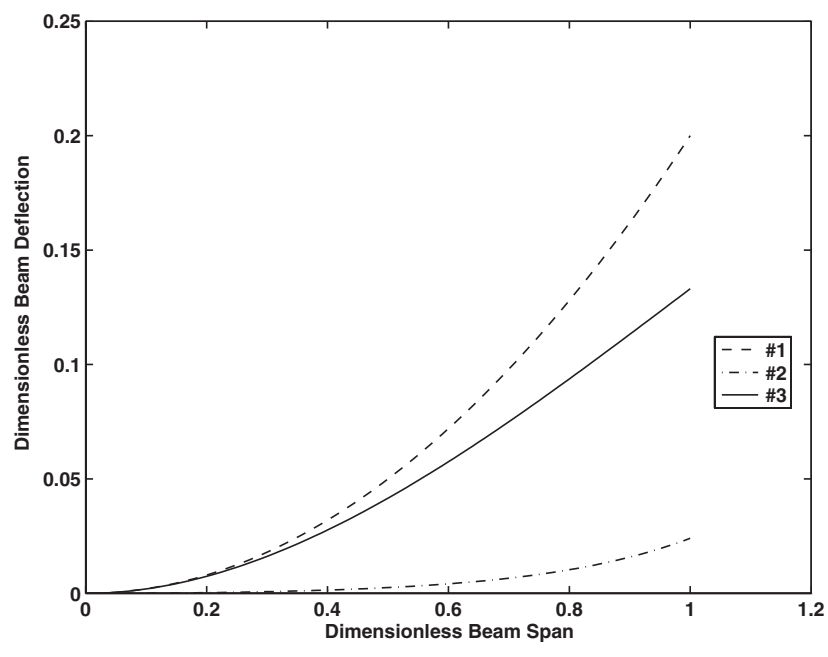

Figure 6. A comparison of the deflections computed from the three different models under large loading scenario at $t / L=0.05$ and $\beta L=4$.

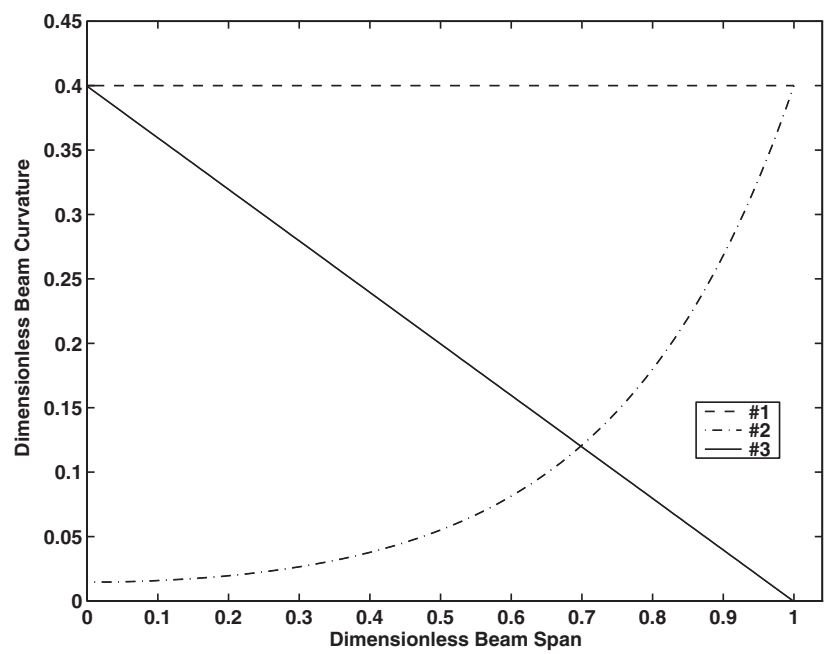

Figure 7. A comparison of the curvatures computed from the three different models under large loading scenario at $t / L=0.05$ and $\beta L=4$.

models of the surface stress effects are presented. As shown, modelling the surface stress effect only as a corresponding concentrated moment at the beam tip, the deflection of the beam is an arc of a circle, which has a uniform curvature. For the other two models, the deflection curvature cannot be maintained a constant. Modelling the surface effect only as a concentrated moment at the beam's free end ignores the stiffening effect of the tensile surface stress and overestimates the beam's deflection. Thus, the curvature of the beam is also overestimated. The stiffening effects of uniformly distributed tensile axial load and concentrated tensile load are also compared. Stoney' formula becomes less accurate as the stiffening effect increases.

\section{Acknowledgments}

This research was supported by the Distinguished Young Scholar Fund of the National Natural Science Foundation 
of China (NSFC, Grant No 10225209), key project from Chinese Academy of Sciences (Grant No KJCX-SW-L2) and NSF-RGC joint project (Grant No 50131160739).

\section{References}

[1] Ibach H 1997 Surf. Sci. Rep. 29193

[2] Schell-Sorokin A J and Tromp R M 1990 Phys. Rev. Lett. 64 1039

[3] Haiss W and Sass J K 1996 Langmuir 124311

[4] Leiva E P M, Del Pópolo M G and Schmickler W 2000 Chem. Phys. Lett. 320393

[5] Fritz J, Baller M K, Lang H P, Strunz T, Meyer E, Güntherodt H J, Gerber Ch and Gimzewski J K 2000 Langmuir 169694

[6] Fritz J, Baller M K, Lang H P, Rothuizen H, Vettiger P, Meyer E, Güntherodt H J, Gerber Ch and Gimzewski J K 2000 Science $\mathbf{2 8 8} 316$

[7] Petelska A and Figaszewski Z A 2000 J. Biophys. 78812

[8] Rasia M and Bollin A 1998 BBA-Biomembranes 1372198

[9] Syms R, Yeatman E M, Bright V M and Whitesides G M 2003 J. Microelectromech. Syst. 12387

[10] Trimmer W S N 1987 Sensors Actuators 19267
[11] Zhao Y P, Wang L S and Yu T X 2003 J. Adhesion Sci. Technol. 17519

[12] Stoney G 1909 Proc. R. Soc. Lond. A 82172

[13] Nix W D 1989 Metall. Trans. A 202217

[14] Hsueh C H 2002 J. Appl. Phys. 919652

[15] Nikishkov G P 2003 J. Appl. Phys. 945333

[16] Klein C A 2000 J. Appl. Phys. 885487

[17] Freund L B, Floro J A and Chason E 1999 Appl. Phys. Lett. 74 1987

[18] Sader J E 2001 J. Appl. Phys. 892911

[19] Godin M, Tabard-Cossa V and Grütter P 2001 Appl. Phys. Lett. 79551

[20] Miyatani T and Fujihira M 1997 J. Appl. Phys. 817099

[21] Liu F, Rugheimer P, Mateeva E, Savage D E and Lagally M G 2002 Nature 416498

[22] Fang W and Wickert J A 1996 J. Micromech. Microeng. 6301

[23] Jaccodine R J and Schlegal W A 1966 J. Appl. Phys. 372429

[24] Press W H, Teukolsky S A, Vetterling W T and Flannery B P 1992 Numerical Recipes in Fortran 2nd edn (Cambridge: Cambridge University Press)

[25] Ren Q and Zhao Y P 2004 Microsystem Technologies 10307

[26] Timoshenko S 1937 Vibration Problems in Engineering 2nd edn (New York: D. Van Nostrand) 